\title{
Determinants of Rational Knots
}

\author{
Louis H. Kauffman ${ }^{1}$ and Pedro Lopes ${ }^{2}$ \\ ${ }^{1}$ Department of Mathematics, Statistics and Computer Science, University of Illinois at Chicago, 851 S. Morgan St., Chicago \\ IL 60607-7045, USA; kauffmanduic . edu \\ ${ }^{2}$ Department of Mathematics, Instituto Superior Técnico, Technical University of Lisbon, Av. Rovisco Pais, 1049-001 Lisbon, \\ Portugal; pelopesemath.ist.utl.pt
}

received November 26, 2007, revised February 10, 2009, June 25, 2009, July 19, 2009, accepted July 12, 2009.

We study the Fox coloring invariants of rational knots. We express the propagation of the colors down the twists of these knots and ultimately the determinant of them with the help of finite increasing sequences whose terms of even order are even and whose terms of odd order are odd.

Keywords: Rational knots, colorings, determinants of knots, checkerboard graphs, spanning trees

\section{Introduction}

A knot is an embedding of the circle into three-dimensional space. Knots that are obtained from one another by continuous deformation of their embeddings are said to be equivalent. The classification of these equivalence classes is still an open problem. In order to study a knot one usually resorts to projecting it into a plane in such a way that the singularities of the projection look locally like the crossing of two line segments. At these crossings the line that goes under in the embedding is broken in the projection giving rise to the so-called knot diagram (see Figure 1).

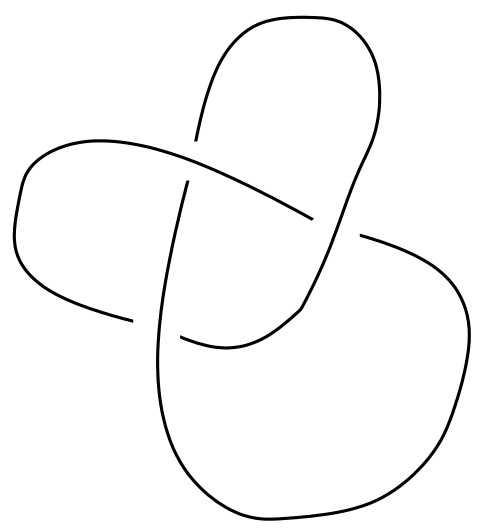

Fig. 1: A diagram of the trefoil knot.

Knot diagrams are planar networks of arcs. The Reidemeister moves ([8]) are defined to be local transformations on the diagrams. The Reidemeister theorem states that, given two diagrams, the knots corresponding to these diagrams are equivalent if and only if these diagrams are obtained from one another by a finite number of the Reidemeister moves. Whenever a mathematical object is associated to a knot diagram in such a way that this object remains the same when Reidemeister moves are performed on the diagram, then this object is called a knot invariant. By Reidemeister's theorem, a knot invariant is an invariant of the equivalent class of the knot. The knot invariant we will be concerned with in this article is called Fox coloring ([8]).

We now elaborate on Fox colorings. Given any knot diagram of the knot under study, the arcs of the diagram stand for algebraic unknowns and the equation "the sum of the under-arcs equals twice the over-arc" is read off at each crossing (see Figure 2). The system of equations so obtained has a matrix of coefficients over the integers. 
The equivalence class of this matrix under elementary operations on matrices (as listed on page 50 of [12]) over the integers constitutes an invariant of the knot under study. The elementary operations on matrices over the integers are standard row and column operations plus enlarging the matrix by a row and column of zeros except for the diagonal entry of 1 , or removing such a row and column.

Other invariants of the knot stemming from this one are the following. The invariant factors of the equivalence class of the matrix; any first minor (also known as the determinant of the knot); the number of solutions of the system of equations over the integers modulo $r$ (also known as the number of Fox $r$-colorings). The minimum number of distinct colors it takes to represent such a nontrivial solution (i.e., a solution such that at least two unknowns take on different values) across the diagrams that represent this knot, is a subtle and interesting invariant ([11]).

Some of these invariants are easy to calculate and, at the same time, seem to be effective enough to be interesting (number of colorings, see [13], [5]). Others are hard to determine and related to elusive conjectures (minima of colors, see [7], [10], [2], [11]).

In [5] a brute force approach was used: a knot diagram was introduced into a computer program that converted it into a coloring system of equations. Then, candidates for solutions of the coloring system of equations were tested. Finally, the actual solutions were counted ([5]).

In [11] the focus was on a particular class of knots and a general formula was obtained for the number of colorings. For this class of knots, a Gaussian reduction of the systems of equations was developed by inspection of the knot diagram under study. Some unknowns were enough to express the rest of them. This led to the development of a black-box approach to diagrams. What is inside a given part of the diagram, the so-called blackbox, is ignored. All that matters is that there is a "color input" and a "color output" to this part of the diagram. By calculating the propagation of the "color input" down the black-box, the "color output" is specified in terms of the "color input" and the "size" of the black-box. Finally we equate this "color output" to the "color input" in order to obtain the coloring system of equations and other invariants like the determinant of the knot (we elucidate this below, see Section 2). This technique can then be applied to more complicated situations. Whenever a knot diagram involves some combination of these black-boxes, we use this approach, thus reducing the number of unknowns and the number of equations we have to work with.

A particular situation comes up with a "twist", which is obtained by twisting two line segments an assigned number of times (see the left-hand side of Figure 3 for a particular twist). Since the propagation of colors down a twist is well-understood (see [11]) and since rational knots (see Section 3 below) can be regarded as special stackings of twists, we look into calculating the propagation of colors down the twists of a rational knot. We denote $R\left(n_{1}, n_{2}, \ldots, n_{N}\right)$ the rational knot with $n_{i}$ crossings on the $i$-th twist. The formulas we obtain involve three polynomials in the number of crossings on each twist, the $n_{i}$ 's. These polynomials are closely related to the set of increasing sequences of integers from $\{1,2, \ldots, N\}$ whose terms of even order are even and whose terms of odd order are odd, call this set $I E O[N]$. The coefficients of these polynomials are all equal to 1 . In each monomial the power of each $n_{i}$ is either 1 or 0 . One of the polynomials, call it $p_{N}$, has as many monomials as there are sequences in $I E O[N]$. As for the other two, one of them, call it $p_{N}^{e}$, is obtained from $p_{N}$ by deleting the monomials which are products of an odd number of $n_{i}$ 's. The other polynomial, call it $p_{N}^{o}$, equals $p_{N}-p_{N}^{e}$. Moreover, the relation between the monomials of $p_{N}$ and the sequences in $I E O[N]$ is as follows. If $\left(u_{1}, u_{2}, \ldots, u_{k}\right) \in I E O[N],(1 \leq$ $k \leq N)$, then $n_{u_{1}} n_{u_{2}} \ldots n_{u_{k}}$ is a monomial in $p_{N}$ (and conversely).

We prove the following result in Section 6.

Theorem 1.1 Given a non-negative integer $I$, consider $2 I+1$ integers $n_{1}, n_{2}, \ldots, n_{2 I}, n_{2 I+1}$.

1. The coloring system of equations of $R\left(n_{1}, n_{2}, \ldots, n_{2 I}\right)$ and its determinant are, respectively

$$
(b-a) p_{2 I}^{e}=0 \quad \text { and } \quad \operatorname{det} R\left(n_{1}, n_{2}, \ldots, n_{2 I}\right)=p_{2 I}^{e} .
$$

2. The coloring system of equations of $R\left(n_{1}, n_{2}, \ldots, n_{2 I}, n_{2 I+1}\right)$ and its determinant are, respectively

$$
(b-a) p_{2 I+1}^{o}=0 \quad \text { and } \quad \operatorname{det} R\left(n_{1}, n_{2}, \ldots, n_{2 I}, n_{2 I+1}\right)=p_{2 I+1}^{o} .
$$

This article is organized as follows. In Section 2 we introduce the background material and the notion of blackbox in the context of knot diagrams and colorings. In Section 3 we introduce the diagrammatics of rational knots. In Section 4 we calculate the coloring equation and the determinant of a few particular rational knots and single out patterns in their expressions which involve the polynomials referred to above. The polynomials are formally introduced in Section 5 and relations among them are proved. In Section 6 we prove Theorem 1.1 stating the form of the coloring equation and the determinant of a rational knot $R\left(n_{1}, \ldots, n_{N}\right)$. In Section 7 we give an alternative 
approach to the determinants of rational knots and links by counting the spanning trees in their checkerboard graphs. This leads to a simple recursion formula for these determinants. The outputs of these formulas are identical to the results of Theorem 1.1

\section{The Black-box Approach}

We introduce some of the objects we will be dealing with in this article.

Definition 2.1 (Twist) A twist is a portion of a diagram which is given by $\sigma_{1}^{n}$ where $\sigma_{1}$ is a standard generator of the braid group on two strands, $B_{2}$ (see [3]). The left hand-side of Figure 3 depicts $\sigma_{1}^{3}$.

Definition 2.2 (Fox Coloring, [6]) A Fox coloring of a knot or tangle diagram is the assignment of integers to the arcs of the diagram such that at each crossing twice the integer assigned to the over-arc equals the sum of the integers assigned to the under-arcs meeting at this crossing, see Figure 2. We equivalently express this equality as

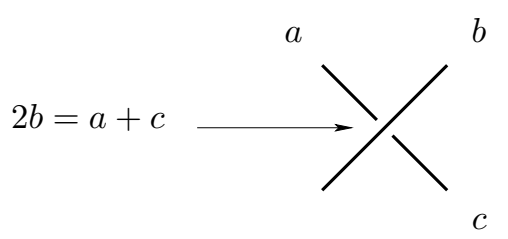

Fig. 2: The equation associated to the unknowns at a crossing.

"the sum of the under-arcs equals twice the over-arc".

Furthermore, these equations and their solutions will be considered modulo $r$ for given integers $r>1$.

In the present work, we do not specify this modulus so that the formulas we obtain are as general as possible. Moreover, in the literature, the modulus is often taken to be a prime number. Here we allow the modulus to take on any composite integer value greater than $2, r \in \mathbb{Z}^{+} \backslash\{1,2\}$.

Definition 2.3 (Coloring System of Equations) A Coloring System of Equations (CSE) of a knot diagram is the system of equations assigned to the knot diagram by regarding the arcs as unknowns and reading the equation $2 b=a+c$ at each crossing (see Figure 2). Usually we solve these CSE's over the integers $\bmod r$, for some modulus $r$. The solutions of this CSE are the Fox colorings of the knot diagram with respect to the specified modulus. The Coloring Matrix (CM) of the knot diagram is the matrix of the coefficients of the CSE of this knot diagram. This is a square matrix since any diagram of a non-trivial knot has as many arcs as crossings. Any matrix obtained from the CM by elementary matrix operations - [12], page 50 - is also known as a coloring matrix. The system of equations associated to this new matrix is also a coloring system of equations for this knot.

Proposition 2.1 Consider a modulus and a knot K. The number of Fox colorings in this modulus for any knot diagram of $K$ is an invariant of the knot. Also, the absolute value of any first minor of the coloring matrix is an invariant of the knot known as the "determinant of the knot" and denoted $\operatorname{det} K$.

Non-trivial colorings occur only for those moduli which are not relatively prime to the determinant of the knot.

Proof: See [8].

Consider the trefoil knot regarded as the closure of $\sigma_{1}^{3}\left(\in B_{2}\right)$ (see left hand-side of Figure 3). Endowing this diagram with a Fox coloring amounts to assigning integers to the arcs of the diagram and making sure that, at each crossing, the rule " $2 b=a+c$ " holds (in a given modulus). On the other hand, working our way down from the top of the braid in the left hand-side of Figure 3, we can regard each crossing as a rule that sets the emerging under-arc equal to twice the over-arc minus the in-coming under-arc.

We believe the assignments to arcs in Figures 3 and 4 to be self-explanatory, Figure 3 for the $n=3$ case and Figure 4 for the general $n$ case (see also [11]). Eventually the bottom arcs are reached and in order to obtain a coloring from this assignment, the color obtained at the bottom right arc has to equal $b$ and the color at the bottom left arc has to equal $a$. These two equations are equivalent to each other and to:

$$
\left\{\begin{array}{l}
n(b-a)=0 \\
0=0 .
\end{array}\right.
$$

The determinant of the trefoil is then 3 , which is the $n=3$ case. We kept here the $0=0$ equation for consistency. In the sequel we will drop it whenever we arrive at a situation where two equations in a system are equivalent. 

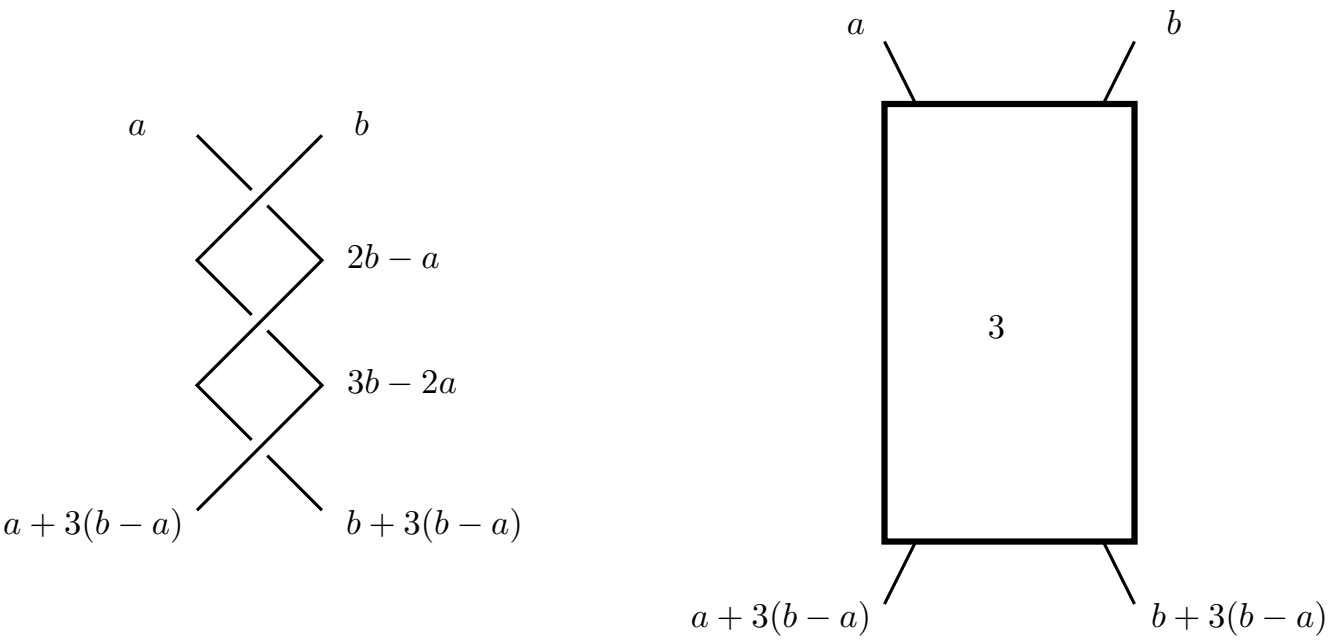

Fig. 3: The braid $\sigma_{1}^{3}$ whose closure yields a diagram of the trefoil, and setting up a Fox coloring. Left: the integral view. Right: the black box approach.

It was not the specifics at each crossing of the braid that allowed us to write down the coloring system of equations (and consequently the determinant of the knot) but the fact that the colors at the bottom strands are expressed in a linear fashion in terms of the colors at the top strands and the number of crossings. This led us to the black-box approach which is depicted on the right-hand side of Figure 3 for the 3 -crossing instance. Here we regard the colors $a$ and $b$ at the top strands as the color input to a black-box labelled with a 3 which transforms them into a color output at the bottom strands, $a+3(b-a)$ and $b+3(b-a)$, from left to right. Figure 4 addresses the case with $n$ crossings.
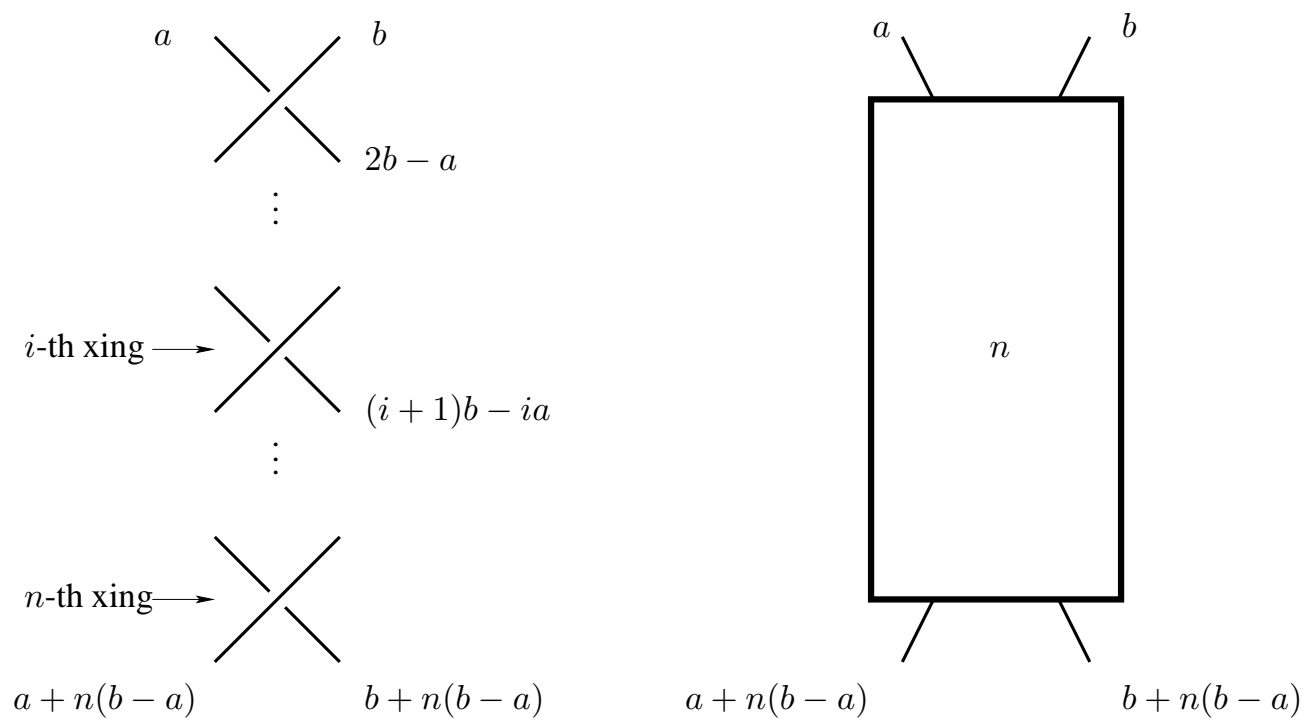

Fig. 4: The braid $\sigma_{1}^{n}$ whose closure yields a diagram of the $T(2, n)$ torus knot, and endowed with a Fox coloring. Left: the integral view. Right: the "black box" approach.

The following Proposition summarizes the situation so far and includes a formula for the calculation of the number of colorings for a $T(2, n)$ knot. A proof can be found in [11].

Proposition 2.2 Given a non-zero integer $n$ and integers $a, b$, and $r(>1)$, consider $\sigma_{1}^{n}\left(\in B_{2}\right)$. Assume a and $b$ is the color input on the top of the braid (see Figure 4). Then the arc emerging from the $n$-th crossing receives color $b+n(b-a)$. The coloring system of equations of the $T(2, n)$ torus knot is formed by $n(b-a)=0 \bmod r$ (and the trivial equation). It has $\operatorname{gcd}(n, r) r$ solutions. Moreover, $\operatorname{det} T(2, n)=n$.

Furthermore, and in view of the preceding discussion, we set forth the following Definition.

Definition 2.4 Consider the right-hand side of Figure 4. It is formed by a rectangular box with two strands on top of it and two strands on the bottom. The box has a label $n$ inside. The top strands are labeled a and $b$ from left to 
right and the bottom strands are labeled $a+n(b-a)$ and $b+n(b-a)$. This ensemble stands for a twist equipped with a Fox-coloring induced by the input $a$ and $b$ on the top. We denote this the Black Box (for the twist) when we ignore the color input. Otherwise we denote it the Black Box (for the twist) endowed with a Fox coloring.

\section{Rational Knots and Their Representation}

In this section we describe the diagrammatic representation of rational knots that fits our needs herein. A formal introduction to this material may be found in [9] and [10].

\subsection{Rational Knots as Special Closures of 4-Strand Braids.}

A rational knot can be regarded as a special composition of twists using the generators $\sigma_{1}$ and $\sigma_{2}$ as well as their inverses, from the braid group on four strands $B_{4}$.

For each positive integer $N$, we choose $N$ integers $n_{1}, n_{2}, \ldots, n_{N}$ and form the braid:

$$
\sigma_{2}^{n_{1}} \sigma_{1}^{n_{2}} \ldots \sigma_{2}^{n_{N-1}} \sigma_{1}^{n_{N}}, \quad \text { for even } N
$$

and

$$
\sigma_{2}^{n_{1}} \sigma_{1}^{n_{2}} \ldots \sigma_{1}^{n_{N-1}} \sigma_{2}^{n_{N}}, \quad \text { for odd } N
$$

See Figure 5 for illustrating examples with $N=4$ and $N=5$ (ignore the broken lines for the moment). We elucidate the sign convention below.

Finally, a special closure of these braids is performed in order to obtain the corresponding rational knot which we have been denoting $R\left(n_{1}, n_{2}, \ldots, n_{N}\right)$. These plat closures are depicted by the broken lines in Figure 5 . We believe Figure 5 is now self-explanatory for the $N=4$ and $N=5$ cases, elucidating the general case. We note that the special closure in the bottom for the even $N$ case (called denominator closure) is different from the odd $N$ case (called numerator closure).

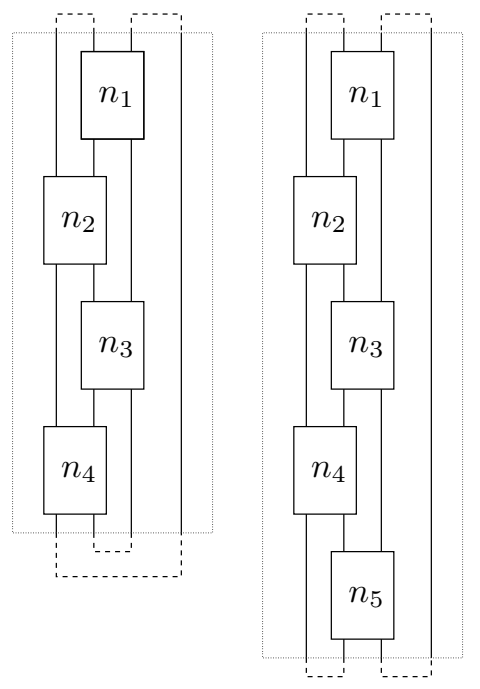

Fig. 5: Rational knots on 4 and 5 twists.

\subsection{Checkerboard shadings and sign convention for crossings}

The signs at crossings we define for diagrams of rational tangles are consistent with the checkerboard shading of knot diagrams in the way defined below.

Definition 3.1 (Checkerboard shadings and sign conventions) A checkerboard shading of a knot diagram is the shading of some regions of the knot diagram such that the four regions meeting at each crossing have the following property. Whenever two of these four regions share a common boundary (which is an arc of the diagram), then one of them is shaded and the other one is not. The signs at crossings are then induced by the checkerboard shading in the following way. Consider a crossing of the diagram along with its over-arc. Rotate this over-arc counterclockwise about the crossing. If the region swept out by the over-arc is the shaded region then the crossing is positive. Otherwise it is negative (see Figure 6 and 7). 


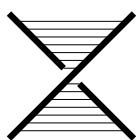

$(+)$

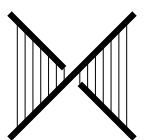

$(-)$

Fig. 6: The signs of the crossings induced by the checkerboard shading.

As a rule, we do not shade the unbounded region around the diagram. See Figure 7 for the illustrative examples of the sign convention and its connection to the checkerboard shadings.
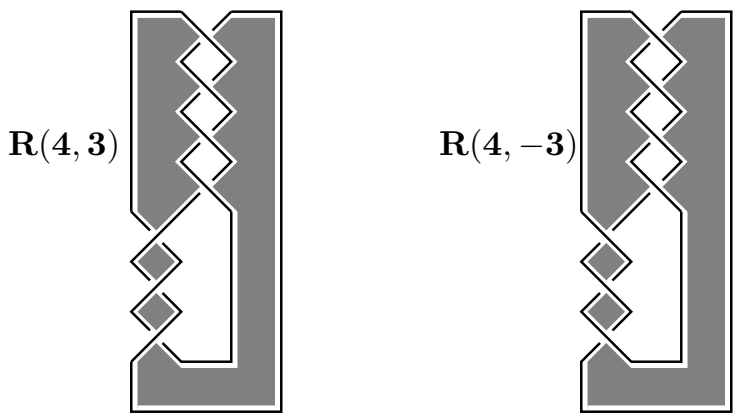

Fig. 7: Sign convention and checkerboard shadings.

\section{The Coloring System of Equations of a Rational Knot: Particular Ex- amples}

In this section we will be always referring to a rational knot with $N$ given twists $(1 \leq N \leq 5)$ where the $i$-th twist has $n_{i}$ crossings, $R\left(n_{1}, n_{2}, \ldots, n_{N}\right)$. The signs of the $n_{i}$ 's are induced by the checkerboard shading of the diagram where the unbounded exterior of the diagram is not shaded, in the way described by Definition 3.1. The general look of the diagrams of these knots that we will be using in the sequel, materializes into the left- and right-hand sides of Figure 5 for $N=4$ and $N=5$, respectively. We usually do not draw the rightmost strand for the rational knots nor do we perform all the plat closures (as we did in Figure 5), in order to graphically bring out the colors the arcs at the bottom are receiving. Nonetheless, the relevant closure of $R\left(n_{1}, n_{2}, \ldots, n_{N}\right)$ is to be assumed. In particular, in Figure 8 the arc assigned $m_{2}$ should be connected to the arc assigned $r_{2}$ and the arc assigned $l_{2}$ should be connected to the arc assigned $a$, following the pattern established in Figure 5 (even case).

\subsection{The rational knots $R\left(n_{1}\right)$ through $R\left(n_{1}, n_{2}, n_{3}, n_{4}, n_{5}\right)$.}

In the next subsections we work out the coloring system of equations and the determinant of the indicated rational knots.

\subsection{1 $R\left(n_{1}\right)$.}

$R\left(n_{1}\right)$ is the torus knot $T\left(2, n_{1}\right)$. As was seen above (Proposition 2.2), the coloring system of equations reduces to:

$$
(b-a) n_{1}=0
$$

and the determinant is

$$
\operatorname{det} R\left(n_{1}\right)=n_{1} \text {. }
$$

\subsection{2 $R\left(n_{1}, n_{2}\right)$}

See Figure 8. Using the black-box approach developed above, we have, at the cross-section $\left(l_{1}, m_{1}, r_{1}\right)$ :

$$
\begin{aligned}
l_{1} & =b, \\
m_{1} & =b+(b-a) n_{1}, \\
r_{1} & =a+(b-a) n_{1},
\end{aligned}
$$




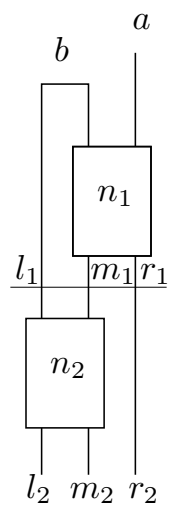

Fig. 8: $R\left(n_{1}, n_{2}\right)$ upon denominator closure.

and so at the $\left(l_{2}, m_{2}, r_{2}\right)$ cross-section we have:

$$
\begin{aligned}
l_{2} & =l_{1}+n_{2}\left(m_{1}-l_{1}\right)=b+(b-a) n_{1} n_{2}=a+(b-a)\left(1+n_{1} n_{2}\right), \\
m_{2} & =m_{1}+n_{2}\left(m_{1}-l_{1}\right)=b+(b-a)\left(n_{1}+n_{1} n_{2}\right)=a+(b-a)\left(1+n_{1}+n_{1} n_{2}\right), \\
r_{2} & =r_{1}=a+(b-a) n_{1} .
\end{aligned}
$$

At this point, we perform a special closure of this rational tangle for even $N$ i.e., we identify the arcs that receive colors $m_{2}$ and $l_{2}$, and the arcs that receive colors $a$ and $l_{2}$. From setting the corresponding pairs of colors equal we obtain two equations which are equivalent to:

$$
(b-a)\left(1+n_{1} n_{2}\right)=0
$$

and thus the determinant of $R\left(n_{1}, n_{2}\right)$ is

$$
\operatorname{det} R\left(n_{1}, n_{2}\right)=1+n_{1} n_{2} .
$$

\subsection{3 $R\left(n_{1}, n_{2}, n_{3}\right)$}

We have (see Figure 9)

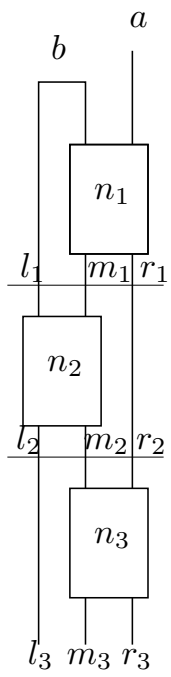

Fig. 9: $R\left(n_{1}, n_{2}, n_{3}\right)$ upon numerator closure.

$$
\begin{aligned}
l_{3} & =l_{2}, \\
m_{3} & =m_{2}+\left(r_{2}-m_{2}\right) n_{3}, \\
r_{3} & =r_{2}+\left(r_{2}-m_{2}\right) n_{3} .
\end{aligned}
$$


Making use of the results obtained in the preceding subsection,

$$
\begin{aligned}
l_{3} & =a+(b-a)\left(1+n_{1} n_{2}\right), \\
m_{3} & =a+(b-a)\left(1+n_{1}+n_{3}+n_{1} n_{2}+n_{1} n_{2} n_{3}\right), \\
r_{3} & =a+(b-a)\left(n_{1}+n_{3}+n_{1} n_{2} n_{3}\right) .
\end{aligned}
$$

We perform a special closure on the tangle in Figure 9 and so the coloring system of equations is here

$$
(b-a)\left(n_{1}+n_{3}+n_{1} n_{2} n_{3}\right)=0,
$$

and

$$
\operatorname{det} R\left(n_{1}, n_{2}, n_{3}\right)=n_{1}+n_{3}+n_{1} n_{2} n_{3} .
$$

4.1.4 R( $\left.n_{1}, n_{2}, n_{3}, n_{4}\right)$

Analogously,

$$
\begin{aligned}
l_{4} & =a+(b-a)\left(1+n_{1} n_{2}+n_{1} n_{4}+n_{3} n_{4}+n_{1} n_{2} n_{3} n_{4}\right), \\
m_{4} & =a+(b-a)\left(1+n_{1}+n_{3}+n_{1} n_{2}+n_{1} n_{4}+n_{3} n_{4}+n_{1} n_{2} n_{3}+n_{1} n_{2} n_{3} n_{4}\right), \\
r_{4} & =a+(b-a)\left(n_{1}+n_{3}+n_{1} n_{2} n_{3}\right) .
\end{aligned}
$$

The coloring system of equations is

$$
(b-a)\left(1+n_{1} n_{2}+n_{1} n_{4}+n_{3} n_{4}+n_{1} n_{2} n_{3} n_{4}\right)=0,
$$

and

$$
\operatorname{det} R\left(n_{1}, n_{2}, n_{3}, n_{4}\right)=1+n_{1} n_{2}+n_{1} n_{4}+n_{3} n_{4}+n_{1} n_{2} n_{3} n_{4} .
$$

4.1.5 $R\left(n_{1}, n_{2}, n_{3}, n_{4}, n_{5}\right)$

And analogously,

$$
\begin{aligned}
l_{5}= & a+(b-a)\left(1+n_{1} n_{2}+n_{1} n_{4}+n_{3} n_{4}+n_{1} n_{2} n_{3} n_{4}\right), \\
m_{5}= & a+(b-a)\left(1+n_{1}+n_{3}+n_{5}+n_{1} n_{2}+n_{1} n_{4}+n_{3} n_{4}\right. \\
& \left.+n_{1} n_{2} n_{3}+n_{1} n_{2} n_{5}+n_{1} n_{4} n_{5}+n_{3} n_{4} n_{5}+n_{1} n_{2} n_{3} n_{4}+n_{1} n_{2} n_{3} n_{4} n_{5}\right), \\
r_{5}= & a+(b-a)\left(n_{1}+n_{3}+n_{5}+n_{1} n_{2} n_{3}+n_{1} n_{2} n_{5}+n_{1} n_{4} n_{5}+n_{3} n_{4} n_{5}+n_{1} n_{2} n_{3} n_{4} n_{5}\right) .
\end{aligned}
$$

The coloring system of equations is

$$
(b-a)\left(n_{1}+n_{3}+n_{5}+n_{1} n_{2} n_{3}+n_{1} n_{2} n_{5}+n_{1} n_{4} n_{5}+n_{3} n_{4} n_{5}+n_{1} n_{2} n_{3} n_{4} n_{5}\right)=0,
$$

and

$\operatorname{det} R\left(n_{1}, n_{2}, n_{3}, n_{4}, n_{5}\right)=n_{1}+n_{3}+n_{5}+n_{1} n_{2} n_{3}+n_{1} n_{2} n_{5}+n_{1} n_{4} n_{5}+n_{3} n_{4} n_{5}+n_{1} n_{2} n_{3} n_{4} n_{5}$.

\section{The $p_{N}, p_{N}^{e}$, and $p_{N}^{o}$ Polynomials}

The following definitions help us understanding the structure of the formulas for the $l_{N}, m_{N}$, and $r_{N}$ obtained in Subsection 4.1.

Definition 5.1 For each positive integer $N$, let $I E O[N]$ stand for the set of increasing sequences of terms from $\{1,2, \ldots, N\}$, whose even terms are even and odd terms are odd, along with the empty sequence (denoted $\emptyset)$.

If $u \in I E O[N]$, say $u=\left(u_{1}, u_{2}, \ldots, u_{k}\right) \quad(1 \leq k \leq N)$, then let

$$
n_{u}:=n_{u_{1}} n_{u_{2}} \ldots n_{u_{k}}
$$

If $u=\emptyset$, then let

$$
n_{u}:=1
$$


For $N=4$ we obtain, besides the empty sequence, $\emptyset$, the sequences:
(1)
(3)
$(1,2)$
$(1,4)$
$(3,4)$
$(1,2,3)$
$(1,2,3,4)$

Definition 5.2 For each positive integer $N$, we set:

$$
\begin{aligned}
& p_{N}:=\sum_{u \in I E O[N]} n_{u} \\
& p_{N}^{e}:=\sum_{u \in I E O[N]}^{\prime} n_{u}
\end{aligned}
$$

where the' denotes deletion of the monomials which are products of an odd number of $n_{i}$ 's; and

$$
p_{N}^{o}:=p_{N}-p_{N}^{e}
$$

For $N=4$ we obtain,

$$
\begin{gathered}
p_{4}=1+n_{1}+n_{3}+n_{1} n_{2}+n_{1} n_{4}+n_{3} n_{4}+n_{1} n_{2} n_{3}+n_{1} n_{2} n_{3} n_{4}, \\
p_{4}^{e}=1+n_{1} n_{2}+n_{1} n_{4}+n_{3} n_{4}+n_{1} n_{2} n_{3} n_{4}, \\
p_{4}^{o}=n_{1}+n_{3}+n_{1} n_{2} n_{3},
\end{gathered}
$$

Moreover, we recognize that these polynomials were obtained in Subsubsection 4.1.4 in the expressions of the $l_{4}, m_{4}$, and $r_{4}$. In particular, we can now write:

$$
\begin{aligned}
l_{4} & =a+(b-a) p_{4}^{e}, \\
m_{4} & =a+(b-a) p_{4}, \\
r_{4} & =a+(b-a) p_{4}^{o} .
\end{aligned}
$$

with the coloring system of equations:

$$
(b-a) p_{4}^{e}=0
$$

and

$$
\operatorname{det} R\left(n_{1}, n_{2}, n_{3}, n_{4}\right)=p_{4}^{e} \text {. }
$$

Analogous relations are now clear between the polynomials of Definition 5.2 and other calculations performed in Subsection 4.1. We prove below that these relations do not depend on the magnitude of $N$, the number of twists in the rational knots. Before we do that we prove recurrence formulas for the these polynomials which will be useful in the sequel.

Lemma 5.1 Given a positive integer $I$, consider the $2 I+1$ variables $n_{1}, n_{2}, \ldots, n_{2 I}$. We keep the notation above concerning the p polynomials.

1.

In particular,

$$
p_{2 I}^{o}=p_{2 I-1}^{o} \quad \text { and } \quad p_{2 I}^{e}=p_{2 I-1}^{e}+n_{2 I} p_{2 I-1}^{o}
$$

2.

In particular,

$$
p_{2 I+1}^{o}=p_{2 I}^{o}+n_{2 I+1} p_{2 I}^{e} \quad \text { and } \quad p_{2 I+1}^{e}=p_{2 I}^{e}
$$

\section{Proof:}

1. It is enough to realize that $I E O[2 I]$ is the union of $I E O[2 I-1]$ with the sequences with an odd number of terms from $I E O[2 I-1]$ augmented with the term $2 I$.

2. Omitted since it is analogous to the proof of 1 . above. 


\section{The Coloring System of Equations of a Rational Knot: The General Case}

In this section we prove Theorem 1.1. In order to do that we first establish the following result.

Proposition 6.1 Given a positive integer $N$, fix $N$ integers, $n_{1}, n_{2}, \ldots, n_{N}$. Consider the rational knot $R\left(n_{1}, n_{2}, \ldots, n_{N}\right)$ given by the special closure of a 4 -braid. Then, keeping the notation above, for any $1 \leq i \leq N$,

$$
\begin{aligned}
l_{i} & =a+(b-a) p_{i}^{e}, \\
m_{i} & =a+(b-a) p_{i}, \\
r_{i} & =a+(b-a) p_{i}^{o} .
\end{aligned}
$$

Proof: By induction on $N$. The preceding examples show us that the proposition is true for several particular cases, from $N=1$ through $N=5$. Assume the statement is true for a given $N=2 I-1$ and consider Figure 10 . Then,

$$
\begin{aligned}
l_{2 I} & =l_{2 I-1}+n_{2 I}\left(m_{2 I-1}-l_{2 I-1}\right)=a+(b-a) p_{2 I-1}^{e}+n_{2 I}\left(a+(b-a) p_{2 I-1}-a-(b-a) p_{2 I-1}^{e}\right) \\
& =a+(b-a) p_{2 I-1}^{e}+n_{2 I}(b-a)\left(p_{2 I-1}-p_{2 I-1}^{e}\right)=a+(b-a) p_{2 I-1}^{e}+n_{2 I}(b-a) p_{2 I-1}^{o} \\
& =a+(b-a)\left(p_{2 I-1}^{e}+n_{2 I} p_{2 I-1}^{o}\right)=a+(b-a) p_{2 I}^{e} .
\end{aligned}
$$

The second equality above is a consequence of the induction hypothesis and the last equality is a consequence of Lemma 5.1.

Analogously,

$$
\begin{aligned}
m_{2 I} & =m_{2 I-1}+n_{2 I}\left(m_{2 I-1}-l_{2 I-1}\right)=a+(b-a) p_{2 I-1}+n_{2 I}\left(a+(b-a) p_{2 I-1}-a-(b-a) p_{2 I-1}^{e}\right) \\
& =a+(b-a) p_{2 I-1}+n_{2 I}(b-a)\left(p_{2 I-1}-p_{2 I-1}^{e}\right)=a+(b-a) p_{2 I-1}+n_{2 I}(b-a) p_{2 I-1}^{o} \\
& =a+(b-a)\left(p_{2 I-1}+n_{2 I} p_{2 I-1}^{o}\right)=a+(b-a) p_{2 I},
\end{aligned}
$$

and clearly,

$$
r_{2 I}=r_{2 I-1}=a+(b-a) p_{2 I-1}^{o}=a+(b-a) p_{2 I}^{o},
$$

using the induction hypothesis and Lemma 5.1.

In a similar way, assuming now the statement is true for $N=2 I$, we obtain,

$$
\begin{aligned}
l_{2 I+1} & =a+(b-a) p_{2 I+1}^{e}, \\
m_{2 I+1} & =a+(b-a) p_{2 I+1}, \\
r_{2 I+1} & =a+(b-a) p_{2 I+1}^{o} .
\end{aligned}
$$

This completes the proof.

Proof: of Theorem 1.1.

We will only prove statement 2 . for 1 . is similar. As a consequence of Proposition 6.1, the coloring system of equations for $R\left(n_{1}, n_{2}, \ldots, n_{2 I}, n_{2 I+1}\right)$ reduces to $a=a+(b-a) p_{2 I+1}^{o}$ which yields at once

$$
(b-a) p_{2 I+1}^{o}=0 \quad \text { and } \quad \operatorname{det} R\left(n_{1}, n_{2}, \ldots, n_{2 I}, n_{2 I+1}\right)=p_{2 I+1}^{o} .
$$

\section{Counting Spanning Trees of the Graphs Induced by the Checker- board Shadings of Rational Knots}

The checkerboard shadings of a knot diagram (Definition 3.1) suggest the definition of a checkerboard graph in the following way.

Definition 7.1 (The checkerboard graph) Given a checkerboard shading of a knot diagram, we associate to it a graph in the following way. The vertices of the graph are the shaded regions of the diagram. There is an edge between two vertices whenever there is a crossing between the corresponding shaded regions. In particular, two vertices may be connected by more than one edge. 


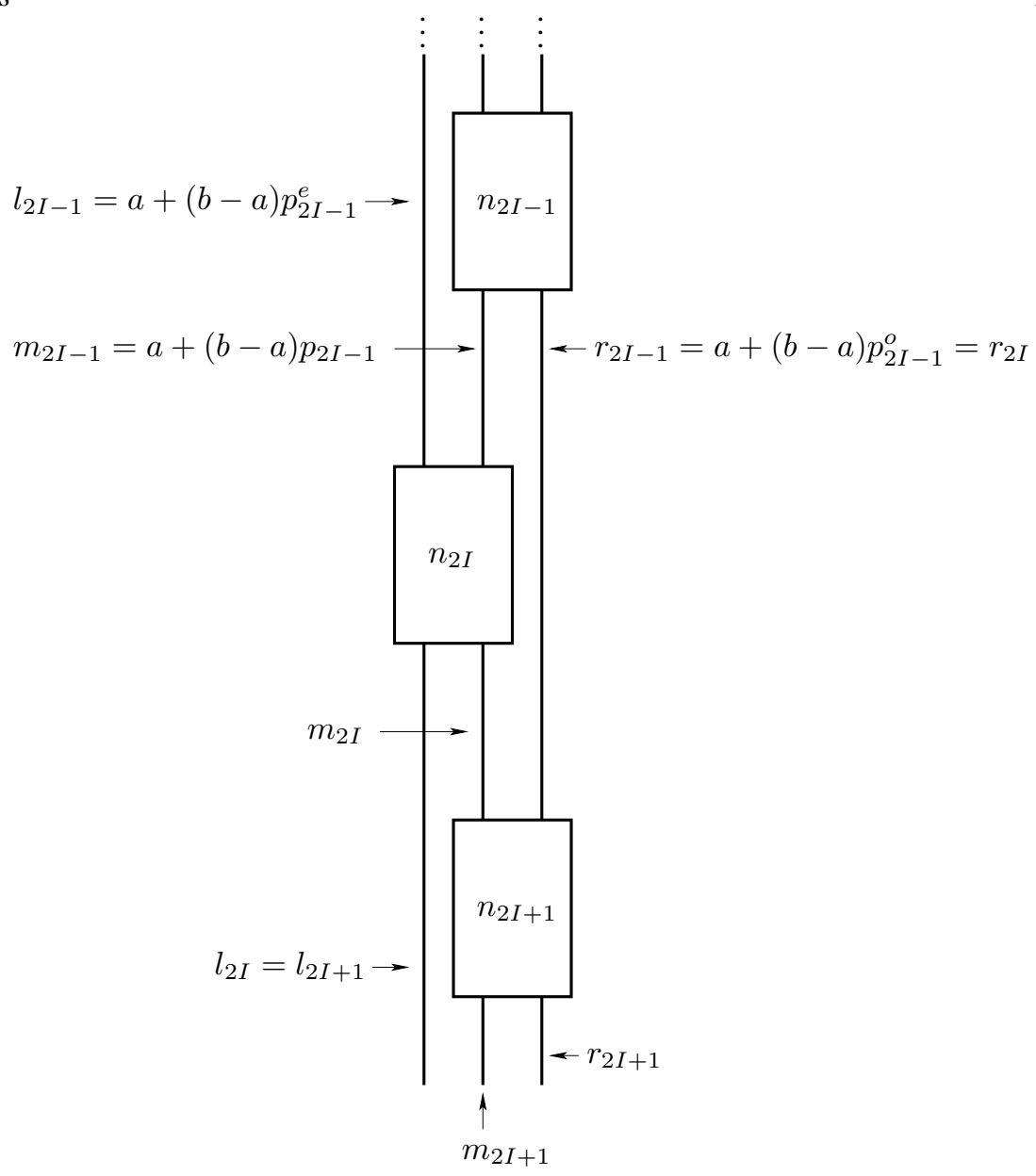

Fig. 10: (Part of) The induction step in Proposition 6.1.

It is a fact that the determinants of alternating knots are given by the counting of the spanning trees of their checkerboard graphs (see [4]). Here we can prove this for rational knots by directly counting the trees and getting the same formula we have derived for rational knots.

We will assume that an infinite sequence of positive integers $\left(n_{1}, n_{2}, n_{3}, \ldots\right)$ has been fixed. Proposition 7.1 below yields a recursive relation for the number of spanning trees as well as a set of instructions to obtain the new ones from the old ones.

Proposition 7.1 Letting $T(N)$ stand for the number of spanning trees of $R\left(n_{1}, n_{2}, \ldots, n_{N}\right)$, then

$$
\begin{gathered}
T(0)=1, \quad T(1)=n_{1}, \\
T(N+1)=n_{N+1} T(N)+T(N-1) .
\end{gathered}
$$

Proof: See Figure 11. In this figure we illustrate how the checkerboard graph is obtained from a diagram of a rational knot, and how the edges in that graph come in either parallel clusters or consecutive sequences depending upon whether the term $n_{N}$ has odd or even index, respectively. With this in mind, one sees that there are two linked formulas for counting the spanning trees in the graph. These formulas are shown in Figure 11. In the figure, the instructions, "delete one", "keep all", "keep one", "delete all" are appended below the terms in the formulas. These are the instructions for forming the spanning trees from the corresponding sequences and clusters. The reader will find this notation self-explanatory. Once both formulas are written down, we see that they can be summarized by the single recursion formula of this proposition. This completes the proof.

Remark 7.1 It is a fact that the determinant of an alternating knot or link is equal to the number of spanning trees in its checkerboard graph. Thus we can state as a corollary to this proposition that $T(N)=D_{N}$ where $D_{N}$ denotes the determinant of the rational knot corresponding to the fixed sequence described above. Thus the formula above gives a recursion formula for the determinants of rational knots and links. The reader will enjoy verifying that this formula yields our previous formulas for these determinants. As a final remark, note that if all the $n_{N}$ equal 1, then the sequence $D_{N}$ is the Fibonacci sequence. 


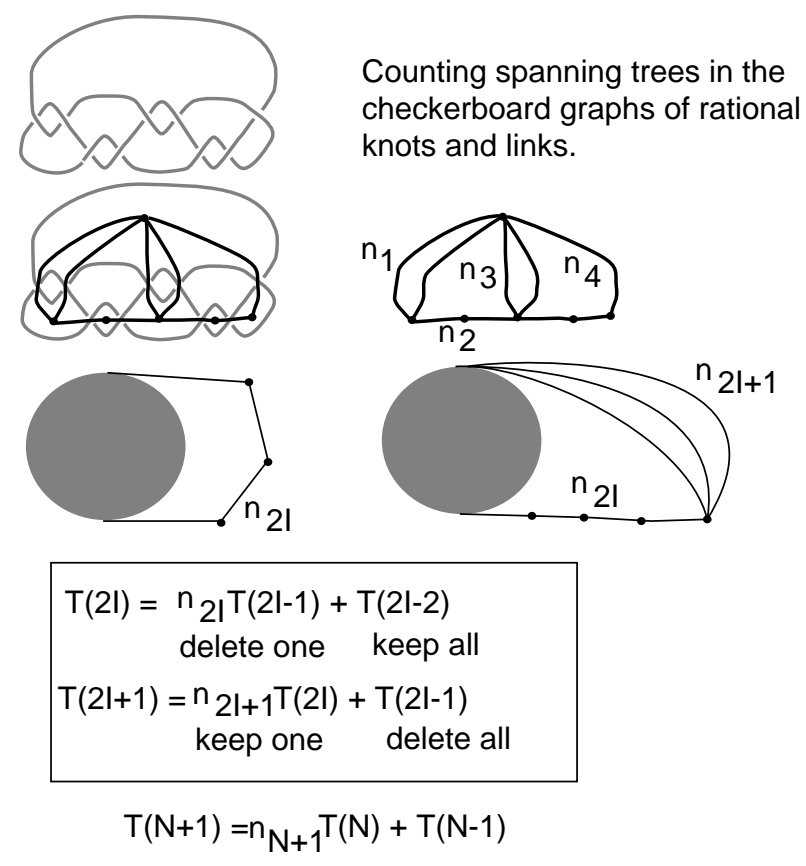

Fig. 11: Tree count

\section{Acknowledgements}

P.L. acknowledges support by Programa Operacional “Ciência, Tecnologia, Inovação” (POCTI) of the Fundação para a Ciência e a Tecnologia (FCT) cofinanced by the European Community fund FEDER. He also thanks the staff at IMPA and especially his host, Marcelo Viana, for hospitality during his stay at this Institution.

We thank the referee for useful suggestions which improved this article.

\section{References}

[1] C. Adams, The Knot Book, W. H. Freeman and Company, New York, (1994)

[2] M. Asaeda, J. Przytycki, A. Sikora, Kauffman-Harary conjecture holds for Montesinos knots, J. Knot Theory Ramifications 13 (2004), no. 4, 467-477

[3] J. Birman, Braids, links, and mapping class groups, Annals of Math. Studies 82, Princeton University Press, Princeton, N. J. (1974)

[4] R. H. Crowell, Genus of alternating link types, Ann. of Math. 69 (1959), 258-275

[5] F. M. Dionísio, P. Lopes, Quandles at finite temperatures II, J. Knot Theory Ramifications, 12 (2003), no. 2, $1041-1092$

[6] R. H. Fox, A quick trip through knot theory, in “Topology of 3-Manifolds and Related Topics" (Georgia, 1961), PrenticeHall (1962) 120-167

[7] F. Harary, L. H. Kauffman, Knots and graphs I. Arc graphs and colorings, Adv. in Appl. Math., 22 (1999), 312-337

[8] L. H. Kauffman, Knots and physics, 3rd edition, Series on Knots and Everything 1, World Scientific Publishing Co., River Edge, NJ (2001)

[9] L. H. Kauffman, S. Lambropoulou, On the classification of rational knots, Enseign. Math., 49 (2003) 357-410

[10] L. H. Kauffman, S. Lambropoulou, On the classification of rational tangles, Adv. in Appl. Math., 33 (2004), no. 2 , 199-237

[11] L. H. Kauffman, P. Lopes, On the minimum number of colors for knots, Adv. in Appl. Math., 40 (2008), no. 1, 36-53

[12] W. B. R. Lickorish, An introduction to knot theory, Graduate Texts in Mathematics 175, Springer Verlag, New York (1997)

[13] P. Lopes, Quandles at finite temperatures I, J. Knot Theory Ramifications, 12 (2003), no. 2, 159-186 